American Journal of Neuroscience 1 (2): 28-33, 2010

ISSN 1948-9900

(C) 2010 Science Publications

\title{
Effects of Increased Physiological Arousal on Upper Extremity Reaction and Movement Times in Healthy Young Adults
}

\author{
Christopher Kovacs and Tamara Bories \\ Department of Kinesiology, Western Illinois University, \\ 220C Brophy Hall, Macomb, IL 61455, Brazil
}

\begin{abstract}
Problem statement: Research has suggested that examining attentional demands during functional tasks is an emergent area of study. Increased arousal may represent an attentional demand, resulting in impaired motor functioning in tasks that require fast reaction and movement times. Approach: This study examined the effects of a non-specific stressor and the resultant physiological arousal on upper extremity functional measures of motor performance. Forty-four young adult participants $(\mathrm{X}$ age $=20.6$ ) were randomly assigned to either a stress/arousal group or non-stress control group. Arousal was altered through the use of the Stroop Color Word Task and mental subtraction tasks. Results: Paired-sample analyses revealed significant differences $(p<.037)$ from pre to post test for measures of reaction time in the stress group. No significant differences were seen for measures of movement time $(\mathrm{p}<.095)$ in the stress group. Conclusion: These results suggest that increased levels of physiological arousal may alter reaction time, movement time and resultant motor functioning in healthy young adults. This increase in physiological arousal may be the result of nonspecific external stressors and have significant implications for movement production accuracy in multiple populations, including older adults. Further research examining this effect in older adults is ongoing.
\end{abstract}

Keywords: Reaction time, physiological arousal, stress group, control group, young adults, motor functioning, significant differences, motor tasks, upper extremity (UE), mental subtraction

\section{INTRODUCTION}

A significant amount of research has examined the dual-task paradigm and its effects on measures of motor performance using a variety of motor tasks (Brown et al., 1999; Maki and McIlroy, 1996). These studies are designed to assess motor performance during the simultaneous performance of cognitive and motor tasks and suggest that increasing attentional demands during the performance of simultaneous tasks have negative effects on one or both of the tasks. These studies, however, have failed to examine the effects of increased physiological arousal on the performance of motor tasks. It may be suggested that increased physiological arousal in the central nervous system during stressful events may act as a secondary task that may lead to decrements in the performance of the primary task. A recent review has suggested that examining attentional demands, such as that which might occur with an increase in physiological arousal, during functional tasks is an emergent area of study and should be extensively examined by investigators in our field (Woollacott and Shumway-Cook, 2002)
Both younger and older adults often demonstrate increased arousal when having to perform sudden, unexpected motor tasks, such as stepping over an obstacle or walking over uneven terrain. In older adults, these anxiety producing events are common and may occur with simple everyday tasks. This increase in physiological arousal may increase the attentional demands during a motor task in a similar manner as that seen during simultaneous task performance. The effects of physiological stress, often associated with arousal levels, have been examined extensively in the literature. However, many of these studies have utilized specific types of external threat to modulate change (Brown et al., 1999; Jones and Hardy, 1988). Although important to examine specific challenges that may increase central nervous system activation, such as walking under conditions of instability, further examination needs to occur regarding the effects of a non-specific threat that may heighten physiological arousal. Much of the everyday activities that may increase stress, or arousal levels in young and older adults alike, are often nonspecific. Often, tasks that require quick reactions and movements to some external stimulus, such as walking

Corresponding Author: Christopher Kovacs, Western Illinois University, Department of Kinesiology,

220C Brophy HallMacomb, IL 61455 Tel: 309-298-1524 Fax: 309-298-2981 
Am. J. Neuroscience 1 (2): 28-33, 2010

through a crowded environment, are affected by simple, everyday stressors that may slightly activate the central nervous system. In addition, tasks such as driving an automobile are often become more difficult under conditions of non-specific central nervous system arousal. Driving an automobile requires an individual to be able to react to external stimuli, such as an unexpected pedestrian or automobile, by moving their upper and lower extremities as rapidly as possible to apply the brake and control the vehicle following the stimulus. Thus, this task can be significantly altered under periods of increased arousal and possibly result in a potentially dangerous event.

Increased physiological arousal is a product of the autonomic nervous system and its response to an increase in stress. Changes in heart rate and blood pressure responses are commonly used to assess the arousal levels of the central nervous system. Additionally, it has been shown that mental challenges also increase the arousal of the CNS, even when paired with concurrent physical activity (Szabo et al., 1994). This suggests that simple mental challenges may be a useful method of increasing non-specific physiological arousal levels in younger adults. This increased activation of the central nervous system may therefore act as a competing "task", thus impairing performance of the first, or primary task, of responding to an external stimulus.

Thus, the goal of this preliminary investigation was to examine the effects if increased physiological arousal in young adults on the performance of reaction and movement time abilities common to different motor skills, such as driving an automobile, walking, or sportrelated activities. Increased physiological arousal was altered through the use of a non-specific "threat" designed to increase the activation of the central nervous system. The researchers hypothesized that increased levels of physiological arousal, produced by an anxiety provoking external event, would result in significant increases in upper extremity reaction and movement times.

\section{MATERIALS AND METHODS}

Participants: Using a pre-test/post-test design, 44 young adult participants with a mean age of $20.6(+/-1.1$ year) were examined. All participants were apparently healthy young adults, with no history of neurological, cardiovascular, or psychological disorders and were at least one year recovered from any orthopedic injuries. No participant was currently on any medication that would alter his or her performance in the study.
Twenty-two participants were randomly assigned to an arousal group that was tested on reaction and movement time measures following an eight-minute stress protocol. Twenty-two participants were also randomly assigned to a control, non-arousal group, which did not receive any stressor and sat quietly for eight minutes between pre-test and post-test measures. Physiological arousal was modified through the use of the Stroop Color Word Task and mental subtraction tasks between pre and post measures. Changes in physiological arousal were measured throughout the protocol using heart rate and blood pressure responses using a Colin Blood pressure Monitor (Colin Corporation, Japan).

Instrumentation: Measurements of reaction time and movement times were assessed using a reaction timer/movement timer (Lafayette Instruments Company, Lafayette, IN). Physiological arousal was assessed using heart rate and blood pressure measures were obtained via a Colin STBP-780 automated blood pressure system (Colin Corporation, Japan). The modified SCWT and mental arithmetic tasks were performed via an Authorware (macromedia, v5.6) program on an IBM 300 GL computer station specifically written for this testing protocol. Data were assessed using Microsoft Excel (Microsoft Corporation, Redmond, WA) and SPSS 10.0 (SPSS Inc., Chicago, IL).

Experimental procedures: Prior to data collection, all procedures were approved by the Institutional Review Board for Ethical Research. All participants signed an IRB approved consent form and completed a medical questionnaire for participation in the study. Participants completed a familiarization session with the researchers. Heart rate and blood pressure were taken for both pre and post stress and reaction time/movement time activities. Eight pre-test trials of RT/MT were collected. During the reaction time tasks, each participant was seated directly in front of the reaction timer. They were instructed to move their dominant Upper Extremity (UE) "as fast as possible" from a button placed to their right to a secondary button placed to the left of their upper torso. Each participant was instructed to move their UE from the left button to the right button the instant a white light appeared on a box placed directly within their line of sight. Each reaction time trial was preceded by a warning light indicating that the primary light would appear within several seconds. This warning light appeared at varying time intervals prior to the primary light for each reaction time trial. 
A stressor task consisted of two minute intervals of alternating SCWT and mental subtraction for a total of eight minutes of mental stress was then performed. The participants were seated in front of a computer monitor and instructed that eight minutes of testing would occur. This consisted of repeating two-minute blocks of the Stroop Color Word Task and mental subtraction. For the Stroop Color Word task, each participant was instructed to depress a key on the computer keyboard that coincided with the font color of a written color that appeared on the computer screen (Ex. The word red might be written in the color blue). This was followed by two minutes of mental subtraction, during which the participant was required to respond to a simple subtraction problem by entering the correct answer using the keyboard (Ex. 299-17 =?). This cycle of two minutes of the SCW and two minutes of mental subtraction was then repeated.

Following completion of the stressor, eight posttest trials of RT/MT were immediately collected within one minute of the mental stressors' completion. All reaction and movement time tasks were counterbalanced to reduce possible learning effects during the data collection. Participants were briefed on the contents and purpose of the study after completion of all testing and any questions were addressed. Averages were calculated of the eight trials for each participant. Group averages were then calculated prior to statistical analysis.

\section{RESULTS}

Reaction/ movement time measures: A one-way repeated measures ANOVA revealed reaction time changes from pre-test to post-test measures were statistically significant $(p=0.037)$ in the arousal group $(p<0.05)$ following the presentation of the stress protocol. Reaction time was slower following the presentation of the stress protocol in the arousal group (Fig. 1). Additionally, movement times were not statistically significant $(\mathrm{p}=0.097)$ from pre-test to posttest measures in the arousal group $(\mathrm{p}<0.05)$ (Fig. 2). Changes in reaction times and movement times were not statistically significant $(\mathrm{p}<0.05)$ from pre-test to post-test measures in the control group.

Physiological measures: A one-way repeated measures ANOVA was calculated comparing the systolic blood pressure of subjects at three different times: Pretest, midtest and posttest. A significant effect was found ( $F$ $=11.2, \mathrm{p}<0.05)$. Follow-up protected t tests revealed that scores increased significantly from pre-test to midtest and decreased significantly from midtest to posttest $(\mathrm{p}<0.01)$ (Fig. 3), suggesting an arousal affect on SBP. A one-way repeated measures ANOVA was also conducted comparing the diastolic blood pressure at pretest, midtest and posttest. A significant effect was found $(\mathrm{F}=25.6, \mathrm{p}<0.05)$. Additional follow-up protected $t$ tests revealed that scores increased significantly from pre-test to mid-test and decreased significantly from midtest to posttest $(\mathrm{p}<0.01)$ (Fig. 4), suggesting an arousal affect on DBP. A one-way repeated measures ANOVA was conducted comparing the heart rate of subjects at pretest, midtest and posttest. A significant effect was found $(\mathrm{F}=37.8, \mathrm{p}<.05)$. Follow-up protected $\mathrm{t}$ tests revealed that scores increased significantly from pre-test to mid-test and decreased significantly from midtest to posttest $(\mathrm{p}<0.01)$ (Fig. 5), suggesting an arousal affect on HR.

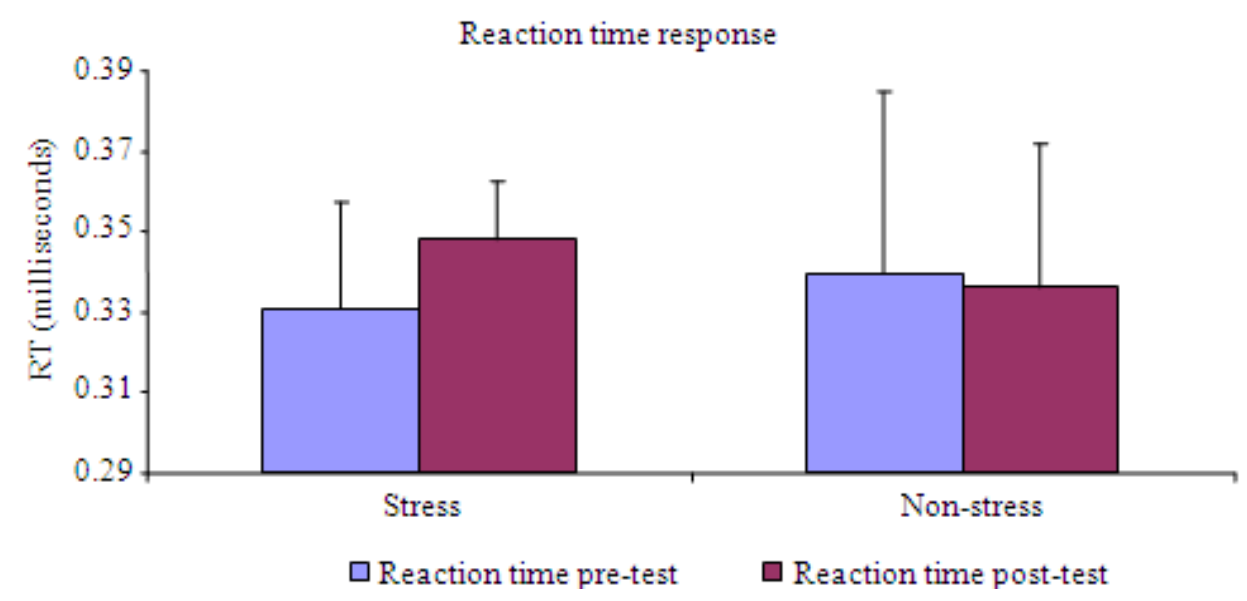

Fig. 1: Reaction time measures between pretest and post-test in both arousal and control groups 
Am. J. Neuroscience 1 (2): 28-33, 2010

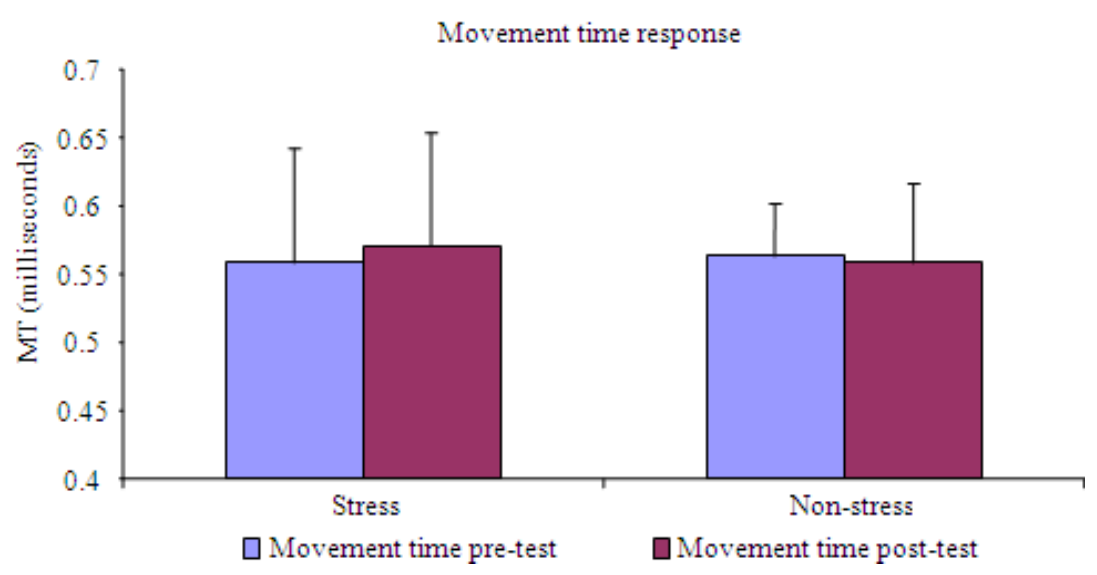

Fig. 2: Movement time measures between pre-test and post-test in both arousal and control groups

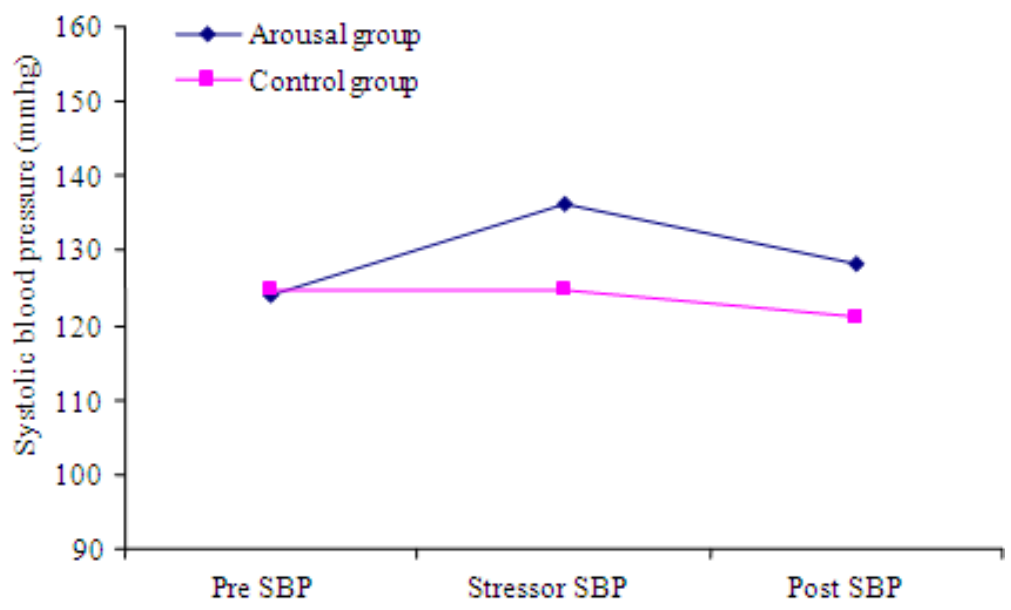

Fig. 3: Systolic blood pressure response between pre-test and post-test in both arousal and control groups

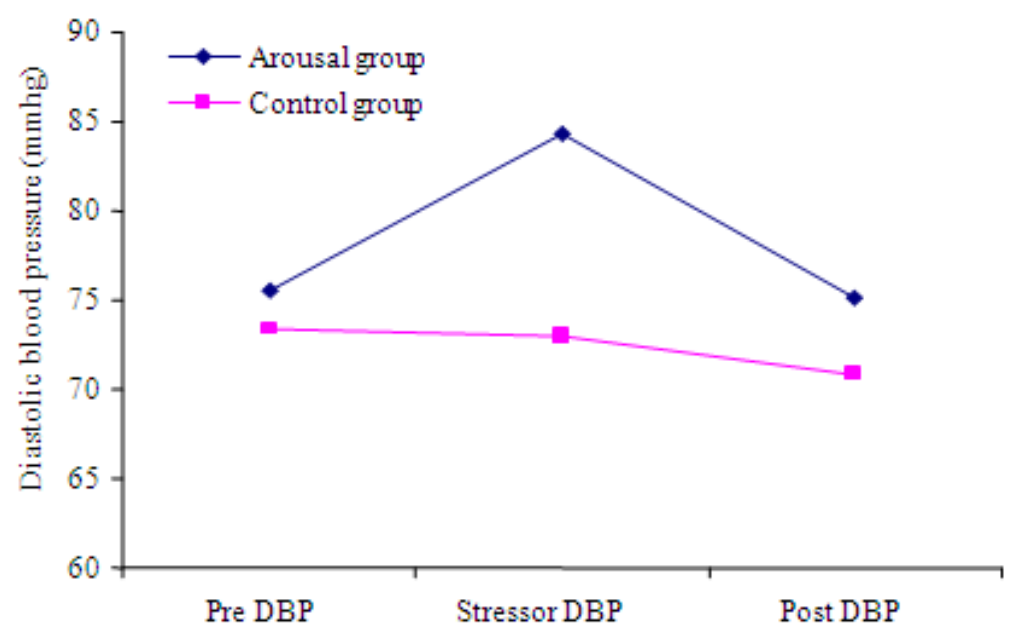

Fig. 4: Diastolic blood pressure response between pre-test and post-test in both arousal and control groups 
Am. J. Neuroscience 1 (2): 28-33, 2010

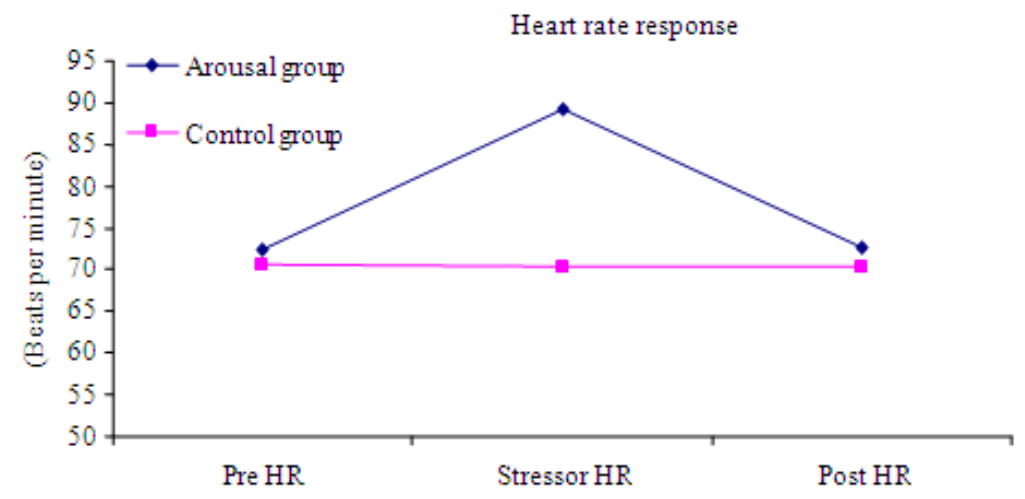

Fig. 5: Heart rate response between pre-test and post-test in both arousal and control groups

\section{DISCUSSION}

These data support earlier investigations of increases in reaction times under conditions of increased arousal (Jones and Hardy, 1988). The goal of our investigation was to examine the effects of nonspecific arousal on reaction and movement times in a young adult population. The data suggest that increasing arousal levels cause a concurrent slowing in reaction times for motor tasks with no significant changes in movement time. These results suggest that neural processing speed in young adults is significantly altered by moderate levels of physiological arousal, while actual movement of the limb is not affected. It may be hypothesized that movement times were not affected due to young adults having significant upper extremity strength, which may help maintain movement times due to this measures direct relationship to muscular action. Earlier research has supported the hypothesis that movement times are not directly affected by an increase in physiological arousal, or anxiety levels (Jones and Hardy, 1988).

Reaction time is a cognitive processing measure that has no relationship to the actual movement production. Increasing levels of arousal may act as a secondary task within the neural network and thus cause an alteration in the performance of the primary movement or task. Further, with a slowing of reaction times in healthy, young adults, tasks requiring fine motor skill and upper extremity movement may be affected. Researchers have suggested that an increase in anxiety may disrupt this conscious processing of information necessary for effective control of human movements and lead to inaccurate movement production by the motor system (Hardy et al., 1996). This may have consequences for both sport-related movements and everyday tasks, such as driving an automobile, which may need to be performed under conditions of increased stress. As attention is diverted from the performance of these skills under conditions of physiological arousal or stress, reactions to changing environmental cues may also be altered, thus increasing the likelihood of an errant movement.

\section{CONCLUSION}

Current research investigations in our lab are examining similar effects in older adults and the degree of reaction time and movement time changes. Changes in both reaction and movement times in an older adult population may have significant effects on activities of daily living and everyday motor tasks, such as driving an automobile. Additionally, it may be beneficial to examine the degree of physiological arousal in older adults, as changes in neural processing may be altered by lowered levels of arousal in older adults when compared to younger adults. Finally, future research should be conducted to examine both lower and upper extremity changes in reaction and movement times to gain a more detailed view of the effects of physiological arousal on total body movements and those tasks that require quick and efficient reactions and movements of the lower extremities.

\section{REFERENCES}

Brown, L.A., A. Shumway-Cook and M.H. Woollacott, 1999. Attentional demands and postural recovery: The effects of aging. J. Gerontol. A Biol. Sci. Med. Sci., 54: 165-171. PMID: 10219006

Hardy, L., R. Mullen and G. Jones, 1996. Knowledge and conscious control of motor actions under stress. Br. J. Psychol., 87: 621-636. PMID: 8962480

Jones, J.G. and L. Hardy, 1988. The effects of anxiety upon psychomotor performance. J. Sports Sci., 6: 59-67. PMID: 3404577

Maki, B.E. and W.E. McIlroy, 1996. Influence of arousal and attention on the control of postural sway. J. Vestibular Res., 6: 53-59. PMID: 8719510 
Szabo, A., F. Peronnet, L. Gauvin and J.J. Furedy, 1994. Mental challenge elicits "additional" increases in heart rate during low and moderate intensity cycling. Int. J. Psychophysiol., 17: 197-204. PMID: 7806464
Woollacott, M. and A. Shumway-Cook, 2002. Attention and the control of posture and gait: A review of an emerging area of research. Gait Posture, 16: 1-14. PMID: 12127181 